\title{
JOURNAL
}

of Health Inequalities

\section{Challenges for the tobacco endgame}

\author{
Mark Parascandola \\ Tobacco Control Research Branch, National Cancer Institute, Rockville, United States
}

ADDRESS FOR CORRESPONDENCE: Mark Parascandola, PhD, MPH, Tobacco Control Research Branch, National Cancer Institute, 9609 Medical Center Drive, Rockville MD 20852,USA, e-mail: paramark@mail.nih.gov

\begin{abstract}
A symposium titled "Preparing for the Tobacco Endgame" was held as part of an international conference organized by the President Stanisław Wojciechowski State University of Applied Sciences in Kalisz, Poland, in June 2019. The international panel of speakers described both how much progress has been made in tobacco control over the past 50 years in Poland, the US, and elsewhere, but also highlighted ongoing challenges. Key challenges include an "implementation gap" for evidence-based tobacco control policies, the complex and dynamic nature of a changing tobacco product market, and ongoing interference of the tobacco industry in tobacco control efforts.
\end{abstract}

KEY WORDS: tobacco control, implementation science, history, tobacco industry.

The subtitle of this session is "Preparing for the Tobacco Endgame". Is the tobacco endgame truly within our grasp? Much progress has been made since the early reports documenting cancer and other health effects from cigarettes in the 1950s and early 1960s. Today, there is an overwhelming volume of evidence about the adverse health effects of cigarette smoking. Smoking is linked to over 40 specific diseases and health conditions and effects almost every organ and system of the body. Decades of experience and evidence demonstrate that comprehensive tobacco control programs can reduce tobacco use and related disease and save lives. A framework for global tobacco control, along with a global community to support it, is provided by the World Health Organisation (WHO) Framework Convention for Tobacco Control (FCTC), and the MPOWER (monitor, protect, offer, warn, enforce, raise) provides an established set of evidence-based tobacco control measures and tools for countries to implement.

However, as the speakers in this session have highlighted, the environment in which tobacco control policies and interventions are implemented is dynamic and changing. This situation creates new and ongoing challenges. I will highlight a few major challenges here:

1. The Implementation Gap: According to the latest WHO Report on the Global Tobacco Epidemic, less than half the world's population is covered by the basic recommended MPOWER strategies. For some measures, the level of protection is especially low. For example, only $10 \%$ of the world's population resides in a country or economic region where tobacco tax policy meets the WHO guidelines. And only $20 \%$ reside where they are protected by comprehensive smoke-free workplace policies. So, despite the fact that we know what works and that there is an international legal framework to support action, these measures fall far short of full implementation.

Recently, "implementation science" has become a popular buzzword in health research. This is the study of methods to promote the systematic uptake of evidence-based interventions into routine medical practice. It has been widely reported that new interventions take, on average, 17 years to be incorporated into routine general practice in health care, and about half of the interventions found to be effective in research studies never make it into practice at all. Historically, this researchto-practice gap has not been the concern of researchers. 
But with greater attention to the cost of health care and efficiency of healthcare delivery, there is now a move to ensure that novel interventions shown to be effective get put into practice more rapidly and widely.

We have a similar implementation gap in tobacco control: a set of proven interventions that are not being put into practice. And even when a law or policy is put in place, it may not be effective if not implemented successfully. Thus, a concerted effort is needed to identify and understand barriers to implementation and how to address them.

2. The tobacco control environment today is more complex than ever before: An expanding range of tobacco and nicotine delivery devices are appearing on the market, including electronic cigarette devices, heatnot-burn cigarettes, and smokeless chewing tobacco products. We lack data on the characteristics, use, and long-term health impacts of novel products. While e-cigarettes have the potential to have a positive impact if they help cigarette smokers to move away from conventional combustible cigarettes, this must be balanced against their potential role in increasing nicotine addiction among youths. Moreover, in some countries (including Poland and the US) regulatory requirements are different for e-cigarettes and other novel products compared with conventional cigarettes. This uncertain legal status adds to the complexity and uncertainty around the longterm public health impact of e-cigarettes and other novel products.

3. The Tobacco Industry: The tobacco industry itself continues to adapt and respond to efforts to control tobacco use and regulate tobacco and nicotine products. In this session we heard about how tobacco control successes in the Ukraine are being threatened by tobacco industry interference in the implementation of tobacco control policies. This story is replicated in many other countries. In particular, low- and middle-income countries (LMICs) governments with limited resources and expertise may be more likely to be influenced by tobacco industry arguments and fears of negative economic consequences of tobacco control. The situation is further complicated by tobacco companies positioning themselves as advocates for harm reduction, through developing and promoting novel nicotine delivery products and through organisations like the Philip Morris supported Foundation for a Smoke-Free World.

4. Increasing tobacco health disparities: While much past experience in tobacco control comes from high-income countries (HICs) (especially North America and Europe), the future of the epidemic is in LMICs. While tobacco use prevalence has been declining in HICs, it has remained constant or is increasing in LMICs. Within countries the evidence consistently shows (with few exceptions) that tobacco use is higher among lower income segments of the population - this is true for HICs as well as LMICs. Moreover, there is a growing divide between HICs and LMICs in how to address the tobacco epidemic. For example, in India where chewing tobacco is the predominant form of tobacco use, talk of e-cigarettes or chewing tobacco as harm-reducing alternatives to cigarettes appears particularly counterproductive. Thus, the "tobacco endgame" may look very different across different countries and environments.

One important lesson from the history of tobacco control is that major norm changes and advances in tobacco control often start at the local level. Local jurisdictions play a key role in leading the way with new interventions. In the US, a great deal of tobacco control happens at the state level; some states and cities have taken the lead in going beyond what is required at the national level, such as in adopting comprehensive smoke-free laws or, in recent proposals, to raise the tobacco purchasing age to 21 years. Thus, action at the local level, including in cities, can have a substantial impact in the long run. This conference, organised by the President Stanisław Wojciechowski State University of Applied Sciences with support from the city of Kalisz, brings together tobacco and health experts from around the world. It is platforms like this one that have been essential in advancing tobacco control in past decades, and the discussions here will likewise have an impact far beyond the city of Kalisz, contributing towards reducing the burden of tobacco use worldwide.

\section{DISCLOSURE}

The author reports no conflict of interest. 